\title{
Recomendaciones para el manejo de la vía aérea en caso sospechoso y/o confirmado con COVID-19. Protocolo del Departamento de Anestesiología Cardiovascular del Instituto Nacional de Cardiología Ignacio Chávez
}

\author{
Recommendations for airway management in a suspected or confirmed case with \\ COVID-19. A protocol of the Department of Cardiovascular Anesthesia of the National \\ Institute of Cardiology Ignacio Chávez
}

Humberto Álvarez-Rosales, Bernardo J. Fernández-Rivera, Raúl Guillén-Rojas, Dania E. Escamilla-Ríos, Rafael Herrera-Elizalde, Esli Telona-Fermán, Carlos Vargas-Trujillo, Pablo Núñez-Trejo, Óscar M. Angulo-Lara, Jesús V. Vela-Roque, Alejandra Minakata-Quiroga y Yazmin Guillén-Dolores* Departamento de Anestesiología Cardiovascular, Instituto Nacional de Cardiología Ignacio Chávez, Ciudad de México, México

\begin{abstract}
Resumen
El nuevo coronavirus SARS-CoV-2 (severe acute respiratory syndrome coronavirus 2), detectado en Wuhan (China), causante de la enfermedad por coronavirus 2019 (COVID-19), que se declaró como pandemia, ha causado más de 19 millones de casos confirmados y más de 700 mil muertes en el mundo. Nuestra institución se reconvirtió a hospital COVID desde principios de abril del 2020, con lo que se desarrollaron protocolos de atención específicos, con el objetivo de mejorar la calidad de atención y seguridad de los pacientes y el personal involucrado en su manejo. El manejo de la vía aérea representa uno de los riesgos más altos de contagio por contacto directo en la generación de aerosoles (intubación orotraqueal, aspiración de secreciones, extubación, resucitación cardiopulmonar, terapia de oxígeno de alto flujo, ventilación no invasiva y ventilación invasiva). Presentamos las recomendaciones actuales para el manejo de la vía aérea, así como un protocolo de manejo paso a paso para llevar a cabo un procedimiento con mayor seguridad basados en la literatura reportada hasta el momento.
\end{abstract}

Palabras clave: Vía aérea. Secuencia rápida. COVID-19.

\begin{abstract}
The new coronavirus SARS-CoV-2 (Severe Acute Respiratory Syndrome Coronavirus 2), detected in Wuhan, China, causes coronavirus disease 2019 (COVID-19), which was declared pandemic, and has caused more than 19 million confirmed cases and more than 700 thousand deaths worldwide. When our institution was converted to COVID's hospital since early April 2020, specific care protocols were developed, with the aim of improving the quality of care and safety of patients and the staff involved in their management. Airway management represents one of the highest risks of direct contact infection with aerosol generation (orotracheal intubation, secretion aspiration, extubation, cardiopulmonary resuscitation, high flow oxygen

Correspondencia:

*Yazmín Guillén-Dolores

E-mail: gudyyazy@ hotmail.com

Fecha de recepción: 11-08-2020

Fecha de aceptación: 06-11-2020 DOI: 10.24875/ACM.20000377

Disponible en internet: 02-12-2020 Arch Cardiol Mex. 2021;91(Supl):95-101 www.archivoscardiologia.com CC BY-NC-ND (http://creativecommons.org/licenses/by-nc-nd/4.0/).
\end{abstract}


therapy, noninvasive ventilation, and invasive ventilation). We present the current recommendations for airway management as well as a step-by-step airway management protocol to carry out a more secure procedure based on the literature reported so far.

Key words: Airway management. Rapid sequence intubation. COVID-19.

\section{Introducción}

El nuevo coronavirus SARS-CoV-2 (Severe Acute Respiratory Syndrome Coronavirus 2), que fue notificado por primera vez en Wuhan (China) el 31 de diciembre del 2019 y es el causante de la enfermedad por coronavirus 2019 (COVID-19), que el 11 de marzo del 2020 la World Health Organization (WHO) declaró como pandémica ${ }^{1}$, ha causado hasta la fecha más de 33 millones de casos confirmados y más de 1 millón de muertes en el mundo².

En México el primer caso reportado fue el 28 de febrero del 2020, desde el 30 de marzo la presidencia declaró «emergencia sanitaria por causa de fuerza mayor" y el 21 de abril se declaró la fase 3 por el incremento del número de casos, se implementó la Jornada Nacional de Sana Distancia y el Plan DN-III-E, así como la reconversión de hospitales para la atención de pacientes con COVID-193. En nuestro país se han detectado más de 700,000 casos confirmados y ha causado más de 70,000 defunciones ${ }^{4}$.

El Instituto Nacional de Cardiología Ignacio Chávez recibió sus primeros pacientes a partir del 11 de abril del 2020, ante el anuncio de que el Instituto Nacional de Enfermedades Respiratorias y el Instituto Nacional de Ciencias Médicas y Nutrición llegaran a su capacidad máxima, lo cual impulsó el desarrollo de protocolos de atención emergentes para casos sospechosos y confirmados con COVID-19 en los múltiples departamentos con el objetivo de brindar una atención con la más alta calidad y con la mayor seguridad para el personal involucrado 5 .

Presentamos las recomendaciones más actuales para el manejo de la vía aérea en pacientes con sospecha o confirmación de COVID-19, así como un protocolo paso a paso para el manejo de la vía aérea con mayor seguridad en estos pacientes, basados en la literatura más relevante reportada hasta el momento. El objetivo fue reducir contagios y proteger al equipo involucrado en este procedimiento de alto riesgo de infección por el contacto directo con la generación de aerosoles (intubación orotraqueal, aspiración de secreciones, extubación, resucitación cardiopulmonar, terapia de oxígeno de alto flujo, ventilación no invasiva y ventilación invasiva) $)^{6}$.

\section{Recomendaciones para el manejo de la vía aérea en caso sospechoso y/o confirmado con COVID-19}

Es sumamente importante una adecuada colocación y retirada del equipo de protección personal (EPP), con la siguiente secuencia.

- Colocación:

1. Quítese todos los objetes personales.

2. Póngase el traje aséptico y botas.

3. Un compañero debe ayudar a supervisar.

4. Higiene de manos $(\mathrm{HM}) \rightarrow$ guantes de nitrilo $\rightarrow$ bata desechable/overall $\rightarrow$ mascarilla facial N95 o $\mathrm{P} 100 \rightarrow$ goggles $\rightarrow$ gorro quirúrgico $\rightarrow$ segundo par de guantes $\rightarrow$ al entrar al cubículo tercer par de guantes y segunda bata.

- Retirada:

1. En el cubículo $\rightarrow \mathrm{HM} \rightarrow$ retirada de tercer par de guantes $\rightarrow \mathrm{HM} \rightarrow$ retirada de segunda bata $\rightarrow \mathrm{HM}$ $\rightarrow$ retirada de segundos guantes $\rightarrow \mathrm{HM} \rightarrow$ retirada de gorro $\rightarrow \mathrm{HM} \rightarrow$ retirada de bata $\rightarrow \mathrm{HM} \rightarrow$ retirada de goggles $\rightarrow \mathrm{HM} \rightarrow$ retirada de mascarilla $\mathrm{N} 95 / \mathrm{P} 100 \rightarrow$ retirada de botas $\rightarrow \mathrm{HM} \rightarrow$ retirada de primeros guantes $\rightarrow \mathrm{HM}^{7}$.

\section{Elaboración de un paquete COVID y uso de una lista de verificación}

El equipo encargado del manejo de la vía aérea en pacientes con sospecha y/o confirmación de COVID-19 debe garantizar una adecuada colocación de su EPP de acuerdo con las recomendaciones de la $\mathrm{WHO}^{7-13}$.

Es necesaria la planeación y elaboración de un plan primario, así como un plan de rescate en caso de falla con el equipo encargado de manejar la vía aérea ${ }^{8,10,11,14}$ (Fig. 1).

Se recomienda el armado previo de bolsas individuales con todo lo necesario para el manejo de la vía aérea en paciente con sospecha y/o confirmación de COVID-19 o la implementación de un carro de vía aérea exclusivo para COVID-198,10,11,13-15.

La lista de verificación se muestra en la tabla 1, se deberá leer en voz alta y tiene que marcarse la confirmación del material, con el objetivo de no olvidar 


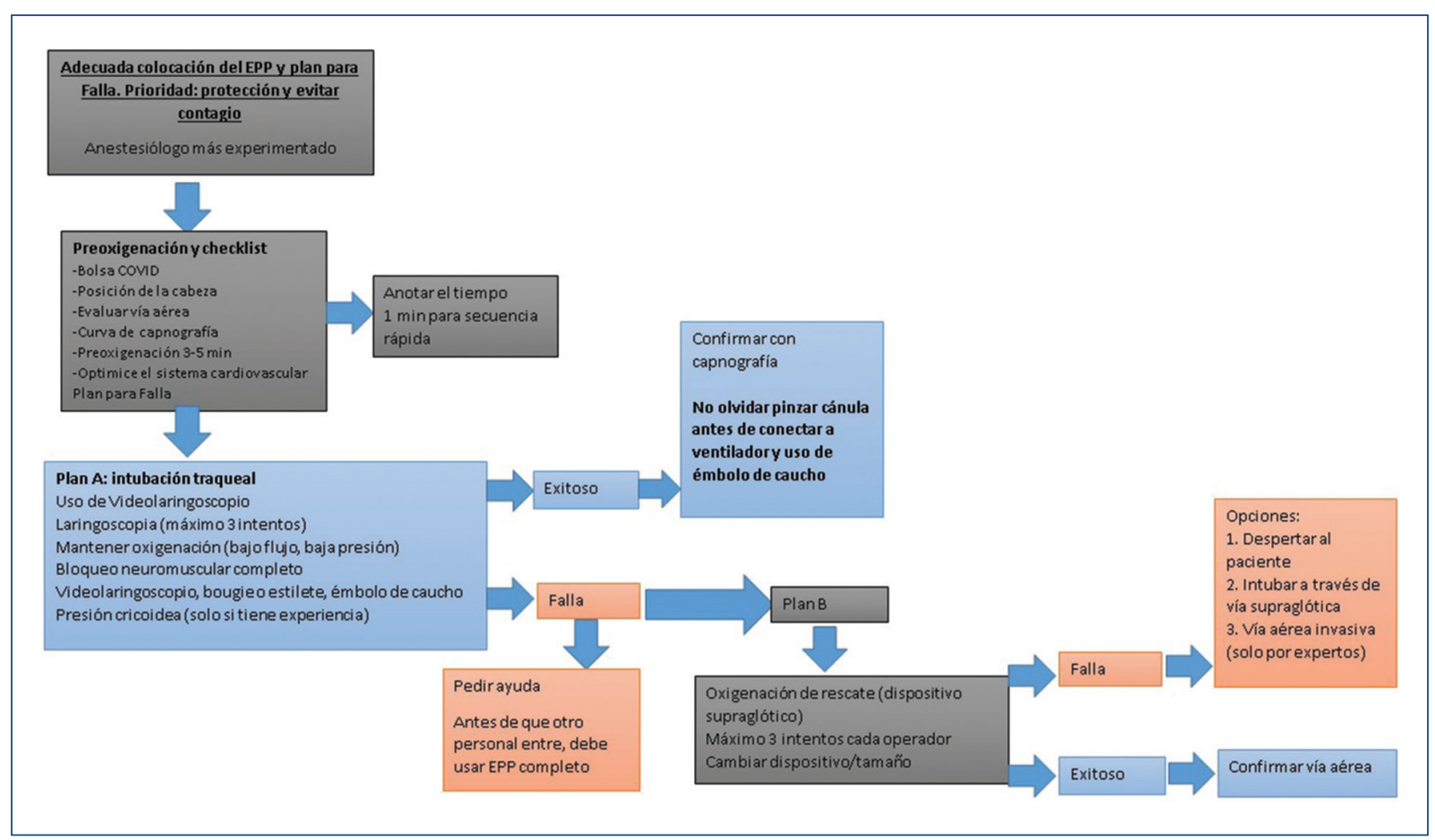

Figura 1. Algoritmo de manejo de vía aérea en paciente con sospecha y/o confirmación de COVID-19. EPP: equipo de protección personal.

ningún elemento. Se debe garantizar una comunicación efectiva en todo momento ${ }^{8}$.

\section{Preparación}

Se recomienda que ingresen el menor número de personas y permanezcan el menor tiempo posible dentro del cubículo o área de intubación ${ }^{8}$, con el objetivo de evitar una exposición innecesaria al virus por parte del equipo ${ }^{10}$. El equipo debe estar conformado por dos o tres personas máximo, se deben asignar roles específicos antes de ingresar a la unidad de intubación, los roles deben ser, por ejemplo: anestesiólogo $A$, anestesiólogo $B$ y/o ayudante, se puede considerar al último como ayudante externo en caso necesario 8 -12,14,16,17.

El paciente debe portar continuamente su cubrebocas durante su estancia en el cubículo, el cual se prefiere sea aislado e idealmente con presión negativa ${ }^{9,14,16,18}$. Se deben optimizar las condiciones del paciente antes de la inducción (bolos de líquidos pequeñas, uso de vasopresores y/o inotrópicos en caso de ser necesario) $)^{14,18}$.

El más experimentado debe realizar la intubación con el objetivo de realizar el manejo de la vía aérea al primer intento ${ }^{8,9,11,12,14,18}$. Se recomienda el uso de técnicas conocidas y familiares para el manejo de la vía aérea ${ }^{8,14}$, el uso de videolaringoscopia debe ser de primera elección, ya que permitirá mantener una mayor distancia con la vía aérea y minimizar la exposición a la generación de aerosoles ${ }^{8-10,12-17}$. En caso de no lograr una intubación al primer intento, se recomienda el uso de dispositivo laríngeo de segunda generación y seguir un algoritmo de rescate ${ }^{10}$.

Se recomienda acomodar la posición del paciente antes de dar inicio al procedimiento, es preferible una posición de rampa o de Trendelemburg invertido en el paciente obeso, con el objetivo de maximizar el tiempo de apnea segura ${ }^{8}$.

Es recomendable el uso de filtros HEPA (high efficiency particulate air) con eficacia del $99.995 \%$ contra partículas virales; estos deben colocarse en el ventilador mecánico a nivel de las válvulas inspiratoria y espiratoria, así como entre la parte distal del circuito del ventilador y la porción distal de la cánula orotraqueal o la porción distal del sistema de succión cerrada-10,13,18. Una compresa húmeda debe cubrir la válvula espiratoria con el objetivo de reducir la exposición de los aerosoles ${ }^{10,15}$. La cánula orotraqueal debe portar una guía en su interior y un sello o tapón en la parte distal de esta antes de utilizarse ${ }^{13}$.

Se recomienda seguir una lista de verificación o un algoritmo para optimizar el tiempo dentro de la unidad de intubación, usar un lenguaje claro y una comunicación 
Tabla 1. Lista de verificación. Las instrucciones son "Leer en voz alta y marcar cada punto» Lista de verificación

3 bolsas (1 para cubrir caja de acrílico y las otras para material de desecho y reutilización)

Sistema Bain con mascarilla facial

Cánulas orotraqueales 7.5 (paciente femenino) y 8 (paciente masculino) con aspiración subglótica

2 filtros de alta eficiencia

Cánula de Yankahuer o Nelaton para aspiración

Estilete o guía

Videolaringoscopio (con todos sus componentes)

Jeringas: 2 de $20 \mathrm{ml}, 3$ de $10 \mathrm{ml}$ y 2 de $5 \mathrm{ml}$; agujas de adecuado calibre

Medicamentos (inductor, relajante neuromuscular, narcótico, lidocaína, atropina, epinefrina)

Tela adhesiva, fijación de cánula orotraqueal

Compresa

Laringoscopio con hoja Macintosh 3 y 4

Cánulas de Guedel 4 (paciente femenino) y 5 (paciente masculino) (90 y $100 \mathrm{~mm}$, respectivamente)

Pinza para tubo o pinza fuerte

Sonda orogástrica (16-18 Fr)

Gel lubricante

Verificar en la unidad de intubación

Aminas vasoactivas y sedación de mantenimiento preparadas

Fijación permanente de cánula orotraqueal

1 caja de acrílico

Ventilador funcionando con filtros HEPA en la válvula inspiratoria y espiratoria.

Asignar roles (anestesiólogo A: intubador; anestesiólogo B: ayudante)

HEPA: high efficiency particulate air.

efectiva, dar instrucciones simples, hablar despacio y repetir las instrucciones por el equipo para verificar el adecuado entendimiento y ejecución de las indicaciones ${ }^{8,18}$.

Los pasos a seguir se muestran en la tabla 2.

\section{Técnica de inducción e intubación}

La inducción debe ser con técnica de secuencia rápida, la presión cricoidea se recomienda solo si el asistente está entrenado, de lo contrario puede contribuir a transformar una vía aérea fácil en difícilo,9,16-18.

La preoxigenación se debe realizar por un tiempo de 3-5 min, con fracción inspirada de oxígeno al 100\%, la mascarilla facial debe sellar adecuadamente y se debe instruir al paciente para que no hiperventile o tome res-
Se recomienda el uso de un circuito cerrado (p. ej., circuito anestésico) o un circuito de reinhalación (circuito Mapleson C, p. ej., sistema BAIN) ${ }^{8}$, si el paciente tiene BIPAP (bilevel positive airway pressure), se recomienda continuarlo durante la fase de preoxigenación para maximizar la oxigenación solo si la mascarilla hace un adecuado sellado a la cara del paciente ${ }^{14}$.

Entre los medicamentos de inducción se debe considerar un inductor, un relajante neuromuscular de acción corta y un opioide. Los medicamentos y dosis a considerar son:

- La ketamina puede considerarse en caso de inestabilidad hemodinámica, las dosis recomendadas son de $1-2 \mathrm{mg} / \mathrm{kg}$ o etomidato 0.1 a $0.2 \mathrm{mg} / \mathrm{kg}^{8}$; si hay mejor estabilidad hemodinámica, propofol $1-2 \mathrm{mg} / \mathrm{kg}^{16}$ o midazolam $0.05 \mathrm{mg} / \mathrm{kg}$. 
Tabla 2. Preparación. Las instrucciones son «Leer la lista de verificación en voz alta y marcar cuando la acción se cumpla"

\section{Lista de verificación}

Verificar existencia de consentimiento informado

Se identifica el equipo por nombre y cargo

Datos del paciente: nombre, edad, peso, talla, comorbilidades, ayuno, ausencia de prótesis dentales

Monitorización del paciente (electrocardiograma, oxímetro de pulso, tensión arterial, capnógrafo disponible)

Compruebe un acceso venoso funcional

Corrija la posición del paciente para la intubación (posición de olfateo, de rampa)

Verifique que el paciente porte cubrebocas y oxígeno suplementario

Coloque una caja de acrílico a la cabeza del paciente

Coloque y fije una bolsa de plástico que cubra la caja de acrílico y al paciente

Anestesiólogo A o intubador

- Introduzca las manos dentro de la caja de acrílico y ya no las retire

Anestesiólogo B

Coloque dentro de la caja lo siguiente:

- Sistema BAIN armado con un filtro HEPA entre la porción distal y la mascarilla facial, una compresa húmeda debe cubrir la válvula espiratoria (objetivo: reducción de aerosol)

- Cánula orotraqueal con una guía metálica a través de un tapón de caucho en la parte distal de la cánula, con una jeringa conectada al globo lista para usarse

- Pinza fuerte o pinza de tubo

- Cánula de Guedel

- Cánula para aspiración con succión cerrada (Huang J)

- Videolaringoscopio con todos sus aditamentos

- 1 bolsa para basura

- 1 bolsa para material esterilizable

- Los medicamentos de la inducción deben estar cargados y rotulados

HEPA: high efficiency particulate air

- Rocuronio como relajante neuromuscular de acción corta a dosis de $1.2 \mathrm{mg} / \mathrm{kg}$ o succinilcolina a dosis de $1.5 \mathrm{mg} / \mathrm{kg}^{8,19}$.

- Fentanilo $2-3 \mathrm{mcg} / \mathrm{kg}$.

- Lidocaína al 2\% 0.5-1 mg/kg ${ }^{16}$.

- El uso de opioides puede ocasionar tos en algunos casos, por lo que se debe considerar este riesgo ${ }^{15}$; se debe prever el uso de vasopresores en caso de hipotensión persistente postinducción ${ }^{8,18,20}$. Las dosis y diluciones recomendadas son las siguientes:

- Fenilefrina en dosis de bolos de 100-200 $\mathrm{mcg}^{20}$.

- Si se requiere soporte hemodinámico adicional:

- Norepinefrina de primera línea, dilución de 80 mg en $250 \mathrm{ml}$ de solución $\mathrm{NaCl} 0.9 \%$ a dosis respuesta para mantener la tensión arterial media (TAM) mayor de $65 \mathrm{mmHg}$.

- Vasopresina, dilución de $40 \mathrm{U}$ en $100 \mathrm{ml}$ de solución $\mathrm{NaCl} 0.9 \%$ a dosis respuesta para mantener TAM mayor de $65 \mathrm{mmHg}$.
Se puede considerar la administración de un anticolinérgico como atropina $(0.5 \mathrm{mg} / \mathrm{kg})$ antes de la inducción para reducir las secreciones en la vía aérea ${ }^{16}$.

Se recomienda el uso de tren de cuatro (TOF) para asegurar una relajación del $100 \%$ en 1 minuto ${ }^{10,13,14}$. Consiste en la aplicación de cuatro estímulos eléctricos separados cada uno por $0.5 \mathrm{~s}$, el resultado se expresa en porcentaje y proporciona un estimado más preciso del bloqueo neuromuscular; el monitoreo puede usarse en la intubación y como un monitoreo durante la estancia en la unidad de cuidados intensivos en caso de uso de relajantes neuromusculares en infusión continua ${ }^{19}$.

No se recomienda la ventilación manual con bolsa mascarilla ${ }^{9}$, sin embargo, si estas se administran deben utilizarse volúmenes tidales y presión de la vía aérea bajos ${ }^{8,9,12}$, y debe ponerse una compresa húmeda alrededor de la boca y nariz del paciente para evitar la aerosolización del virus ${ }^{18}$. En caso necesario, se considera usar una cánula de Guedel para mantener 
Tabla 3. Inducción e intubación adaptado a dos operadores. Las instrucciones son «Puede marcar esta hoja o decir "listo" después de cada acción, asegure comunicación efectiva”

\begin{tabular}{|l|l|l|}
\hline Operador & Acción & Marcar \\
\hline Anestesiólogo B & Informe que inicia procedimiento de inducción e intubación \\
\hline Anestesiólogo A & Retire cubrebocas, suspenda oxígeno suplementario \\
\hline Anestesiólogo B & Conecte el suministro de oxígeno al sistema Bain \\
\hline Anestesiólogo A & Preoxigene 5 minutos con mascarilla facial, cuide el adecuado sellado de esta \\
\hline Anestesiólogo B & $\begin{array}{l}\text { A la indicación del anestesiólogo A administre medicamentos en el siguiente orden: fentanilo } 2 \\
\text { mcg/kg, lidocaína 1 mg/kg, rocuronio } 1.5 \mathrm{mg} / \mathrm{kg} \text { y propofol 1 mg/kg }\end{array}$ \\
\hline Anestesiólogo B & \begin{tabular}{l} 
Cuente 1 minuto, avise y cierre suministro de oxígeno del sistema Bain \\
\hline Anestesiólogo A
\end{tabular} & Realice laringoscopia e informe cuando pase la cánula las cuerdas vocales \\
\hline Anestesiólogo B & Infle el globo y retire la guía lentamente, con cuidado de no retirar el émbolo \\
\hline Anestesiólogo A & Pince el tubo endotraqueal antes de que se retire todo el estilete \\
\hline Anestesiólogo B & Retire el filtro del Bain, colóquelo al tubo endotraqueal, conecte el ventilador y despince el tubo \\
\hline Ambos anestesiólogos & $\begin{array}{l}\text { Verifiquen correcta intubación con capnografía, evalúen signos vitales y optimización } \\
\text { hemodinámica }\end{array}$ \\
\hline
\end{tabular}

permeable la vía aérea una vez que el paciente pierda el estado de consciencia y evitar un cuadro de tos con incremento del riesgo de aerosolización ${ }^{8,14}$.

El tubo orotraqueal debe estar sellado con un émbolo en la parte distal de este y pasar a través del mismo un estilete o bougie ${ }^{8}$; inmediatamente después de la intubación traqueal se debe inflar el globo con aire a una presión entre $20-30 \mathrm{cmH}_{2} \mathrm{O}$, al retirar el estilete se debe evitar la diseminación de secreciones con ayuda de un pinzamiento de la cánula orotraqueal antes de la salida completa del estilete 8,18 .

Para la confirmación de la intubación traqueal se recomienda la verificación de la onda de capnografía y la observación de la expansión bilateral del tórax, no se recomienda la auscultación ${ }^{8,18}$.

Si hay falla a la intubación, de forma alternativa puede usarse un dispositivo supraglótico de 2. a generación, el cual puede ser insertado para ventilar a través de él y reemplazar el rol de la ventilación bolsa-mascarilla con el objetivo de reducir la generación de aerosol debido a mejoría del sello de la vía aérea ${ }^{8,14,18}$.

Se recomienda el uso de barreras para la intubación como el aerosol box, el cual consiste en un cubo de plástico trasparente designado para cubrir la cabeza del paciente con dos puertos designados para el acceso de los brazos para realizar el procedimiento de manejo de vía aérea, en un estudio de simulación publicado en $\mathrm{New}$ England Journal of Medicine se observa que la generación de aerosoles del paciente sin el uso de esta barrera contamina cara y cuerpo del operador, además del cubículo con un radio de hasta 2 metros de distancia; con el uso de la barrera aerosol box solo se contaminó la parte interna de esta, así como los guantes y la parte proximal de los brazos del operador dentro de la barrera, por lo que se sugiere que una barrera de este tipo podría reducir la contaminación del cubículo y reducir el riesgo de contagio del operador que maneja la vía aérea, sin embargo requiere entrenamiento previo, ya que se reduce la movilidad de los brazos y puede incrementar el tiempo de intubación ${ }^{21}$.

La secuencia de pasos de intubación se muestra en la tabla 3.

\section{Fijación y desecho}

Al finalizar el procedimiento se recomienda fijar la cánula orotraqueal con un sistema seguro e iniciar con la separación y desecho del material de forma correcta para evitar contaminación. Se debe colocar en una bolsa la basura o material desechable y el equipo de reutilización debe ser colocado en una segunda bolsa diferente de la anterior ${ }^{14,18}$; se recomienda rociar con una solución desinfectante a ambos materiales (desecho y reutilización) antes de sacarlo del cubículo o área de intubación ${ }^{14,18}$. Los componentes de reutilización deben ser limpiados con solución clorada o peróxido de hidrógeno y/o utilizar 
procesos de esterilización de acuerdo con los protocolos de cada institución, se debe esperar al menos 24 horas antes de volver a usar de forma exclusiva para pacientes con sospecha y/o confirmación de COVID-1914,16,18.

La solución desinfectante puede elaborarse con hipoclorito de sodio en concentración recomendada del $5 \%$ (se debe diluir $100 \mathrm{ml}$ de hipoclorito de sodio en $900 \mathrm{ml}$ de agua), la solución debe prepararse diariamente, ser utilizada en un envase exclusivo (el cual debe rotularse con la concentración de la solución de forma clara) y no debe ser mezclada con jabones u otros limpiadores, de acuerdo con las recomendaciones de la WHO y la PAHO (Organización Panamericana de la Salud) ${ }^{22}$.

\section{Conclusión}

Es necesaria la minimización del riesgo para todo el equipo involucrado en el manejo de estos pacientes. Todos los servicios corren un riesgo alto ante la exposición a la generación de aerosoles, que si se sigue un protocolo de forma sistematizada por el equipo de trabajo el desenlace de contagios podrá reducirse. Es necesario un trabajo en equipo que garantice la mayor seguridad tanto del paciente como del personal y garantizar el mayor bienestar del paciente.

\section{Agradecimientos}

Agradecemos enormemente a nuestros residentes Adriana Moya-Michelena, Arturo Vázquez-Peralta, Candy A. Nicolás-Cortes, Dassaed Serrano-Rivas, Félix A. Rosales-Tovar, José A. Fernández-Vladimir, Karen A. Infante-Sánchez, Laura Ruíz-Torres, Lesly Gonzaliaz-Sánchez, Ligia González-López, Luis F. Ríos-Cuellar y Nora C. Cruz-Rodríguez.

\section{Financiamiento}

Ninguno.

\section{Conflicto de intereses}

Declaramos que no tenemos ningún conflicto de intereses.

\section{Responsabilidades éticas}

Protección de personas y animales. Los autores declaran que para esta investigación no se han realizado experimentos en seres humanos ni en animales.
Confidencialidad de los datos. Los autores declaran que en este artículo no aparecen datos de pacientes.

Derecho a la privacidad y consentimiento informado. Los autores declaran que en este artículo no aparecen datos de pacientes.

\section{Bibliografía}

1. Organización Panamericana de la Salud/Organización Mundial de la Salud. Actualización Epidemiológica: Enfermedad por coronavirus (COVID-19). 20 de abril de 2020, Washington D.C.: OPS/OMS; 2020.

2. COVID-19 Dashboard by the center for systems Science and Engineering (CSSE) at Johns Hopkins University (JHU) [Internet]. Johns Hopkins University of Medicine, Coronavirus Resource Center [fecha de consulta: 29/09/2020]. Disponible en: https://coronavirus.jhu.edu/map.html

3. Aviso epidemiológico - Casos de infección respiratoria asociados a Coronavirus (COVID-19) [Internet]. Gobierno de México, Dirección General de Epidemiología; 10 de abril de 2020. Disponible en: https://www.gob. $\mathrm{mx} /$ salud/documentos/aviso-epidemiologico-casos-de-infeccion-respiratoria-asociados-a-nuevo-coronavirus-2019-ncov

4. Covid-19 México. Información General [Internet]. Gobierno de México [fecha de consulta: 29/09/2020]. Disponible en: https://coronavirus.gob.mx/datos/

5. Comunicados oficiales Instituto Nacional de cardiología Ignacio Chávez [Internet]. Gobierno de México [fecha de consulta: 01/08/2020]. Disponible en: https://www.cardiologia.org.mx/

6. Zhang HF, Bo L, Lin Y, Li FX, Sun S, Lin HB, et al. Response of chinese anesthesiologists to the COVID-19 outbreak. Anesthesiology. 2020;132(6):1333-8.

7. COVID-19: Cómo ponerse y quitarse el equipo de protección personal (EPP) [Internet] World Health Organization [fecha de consulta: 01/07/2020]. Disponible en: https://openwho.org/courses/IPC-PPE-ES

8. Cook TM, ElaBoghdadly K, McGuire B, McNarry AF, Patel A, Higgs A. Consensus guidelines for managing the airway in patients with COVID-19: Guidelines from the Difficult Airway Society, the Association of Anaesthetists the Intensive Care Society, the Faculty of Intensive Care Medicine and the Royal College of Anaesthetists. Anaesthesia. 2020;75(6):785-99.

9. Peng PWH, Ho PL, Hota SS. Outbreak of a new coronavirus: what anaesthetists should know. Br J Anaesth. 2020;124(5):497-501.

10. Yang M, Dong H, Lu Z. Role of anaesthesiologists during the COVID-19 outbreak in China. Br J Anaesth. 2020;124:666-9.

11. Odor PM, Neun M, Bampoe S, Clark S, Heaton D, Hoogenboom EM, et al. Anaesthesia and COVID-19: infection control. Br J Anaesth. 2020;125(1):16-24.

12. Orser BA. Recommendations for Endotracheal Intubation of COVID-19 Patients. Anesth Analg. 2020;130(5):1109-10.

13. Lopez RA, Anthony A, Zuo L, Enomoto TM, Aziz MF. Your COVID-19 intubation kit. Anesth Analg. 2020;131(1):e28-e30.

14. Meng L, Qiu H, Wan L, Ai Y, Xue Z, Guo Q, et al. Intubation and ventilation amid the COVID-19 outbreak: Wuhan's experience. Anesthesiology. 2020;132(6):1317-32.

15. Li W, Huang J, Guo X, Zhao J, Mandell MS. Anesthesia management and perioperative infection control in patients with the novel coronavirus. J Cardiothorac Vasc Anesth. 2020:S1053-0770(20)30284-6. doi: 10.1053/j.jvca.2020.03.035. Online ahead of print.

16. Huang J, Zeng J, Xie M, Huang X, Wei X, Pan L. How to perform tracheal intubation for COVID-19 patients safely and effectively: Experience AND tips from Sichuan, China. J Clin Anesth. 2020;64:109800.

17. Yao W, Wang $T$, Jiang B, Gao F, Wang L, Zheng H, et al. Emergency tracheal intubation in 202 patients with COVID-19 in Wuhan, China: lessons learnt and international expert recommendations. Br J Anaesth. 2020;125(1):e28-e37.

18. London M. Coronavirus disease 2019 (COVID-19): Anesthetic concerns, including airway management and infection control [Internet]. UpToDate; 2020 [fecha de consulta: 15/08/2020]. Disponible en: https://www.uptodate.com/contents/coronavirus-disease-2019-covid-19-airway-management-anesthesia-machine-ventilation-and-anesthetic-care.

19. Rubulotta F, Soliman-Aboumarie H, Filbey K, Geldner G, Kuck K, Ganau $\mathrm{M}$, Hemmerling TM. Neuromuscular blockade monitoring during the COVID-19 Pandemic. Anesth Analg. 2020;9 [Epub ahead of print].

20. Ghia S, Lazar M, Epstein J, Bhatt HV. Anesthesia Stat to Intubate a Coronavirus Disease 2019 (COVID-19) Patient: Implications for the Anesthesiologist. J Cardiothorac Vasc Anesth. 2020;34(10):2566-70.

21. Canelli R, Connor CW, Gonzalez M, Nozari A, Ortega R. Barrier Enclosure during Endotracheal Intubation. N Engl J Med. 2020;382(20):1957-8.

22. Organización Panamericana de la Salud/Organización Mundial de la Salud. Recomendaciones para la preparación de soluciones de desinfección. OPS/CDE/CE/COVID-19/20-0019. 